Polymer Journal, Vol. 38, No. 11, pp. 1105-1113 (2006)

(C) 2006 The Society of Polymer Science, Japan

\title{
Structure and Properties of Novel Fibril Silicate/Rubber Nanocomposites
}

\author{
Ming TIAn, ${ }^{1}$ Yonglai Lu, ${ }^{1}$ Wenli LiAng, ${ }^{1}$ Lijun Cheng, ${ }^{2}$ and Liqun ZHAng ${ }^{2, \dagger}$ \\ ${ }^{1}$ Key Laboratory for nano-materials, Ministry of Education, Beijing 100029, China \\ ${ }^{2}$ The Key Laboratory of Beijing City on Preparation and Processing \\ of Novel Polymer Materials, Beijing 100029, China
}

(Received February 7, 2006; Accepted July 25, 2006; Published October 4, 2006)

\begin{abstract}
Palygorskite (AT) mineral was selected as a nano-fiber precursor due to its unique structure characteristics and surface chemical property, to construct a novel nano-fiber/rubber nanocomposites by using a simple and cost-efficient preparation method. Upon shear force during traditional mechanical mixing, the numerous nano-fibers contained in palygorskite micro-powder were released into rubber matrix resulted from weak stacking force between nano-fibers and high shear stress associated with high viscosity of rubber matrix. Meanwhile these nano-fibers were orientated along the shear direction the same as micro-short fiber. In situ modification using silane coupling agent can improve the dispersion of AT and strengthen the interfacial bonding between AT and rubber. The result from dynamic mechanical thermal analysis shows that the incorporation of palygorskite into rubber matrix markedly lowers the loss factor of rubber in glassy transition region and increases storage modulus of rubber. These nanocomposites exhibit stress-strain characteristics that are similar to that of micro-short fiber reinforced rubber, evident anisotropy in mechanical properties, good processing properties, as well as low cost and easily practiced by industry.
\end{abstract}

[doi:10.1295/polymj.PJ2005231]

KEY WORDS Palygorskite / Nano-fiber / Nanocomposites / Rubber /

Polymer Layered Silicate Nanocomposites (namely PLSNs) have attracted intensive interests from researchers not only in science but also in engineering in the last decades. ${ }^{1-4}$ PLSNs bloomed the research of polymer nanocomposites, and let people realize that nano-compounding dispersions with polymer matrices could endow neat polymer materials with many novel and excellent properties, which originated from the novel nano-compounding structure. Compared with short fiber with micro-diameter reinforced polymer composites, PLSNs exhibited lower density, much higher barrier properties, better product appearance and ability in processing of delicate and complex polymer product. Today, some PLSNs have been commercially produced. ${ }^{5}$

Since the interactions between layers of layered silicate are very strong, ${ }^{1}$ it is not easy to prepare exfoliated PLSNs, in another word, most of PLSNs are intercalated structure, which partially lost the inherent advantages of PLSNs. ${ }^{6}$ These strong layer-layer interactions also make the PLSNs with a higher cost owing to the difficulties in getting nano-dispersion struture.

Theoretically, if the precursor of nano-hard dispersion phase with easily dispersing capability was found, the new polymer nanocomposites should be readily constructed with low cost of preparation. Generally, when the interactions between nano-units included in the precursor are weak, such as van der
Waals force or hydrogen bond rather than strong ionic bonding between layers in layered silicate, it should facilitate the preparation of polymer nanocomposites. Naturally, scientists turned their attentions to those short nano-fibers and expected to make the nanofibers/polymer nanocomposites by realizing a fact that the interaction between nano-fibers is weak van der Waals force. ${ }^{7-11}$

Nano-fiber reinforced polymer materials were early started by Ajayan et al., ${ }^{7}$ and then most of them concentrated on the nano-carbon-fiber or nano-carbon tube/polymer nanocomposites. ${ }^{7-11}$ However, formation and homogeneous dispersion of the fiber-liked nano-phase in polymer matrix is a very challenging work all the time. Compared with in situ polymerization method, ${ }^{12}$ and co-precipitation method from mixing solution, ${ }^{11,13}$ latex compounding method seems to be practically promising for dispersion of carbon nano-tubes in polymer matrix. ${ }^{14}$ Carbon nanotubes and carbon nano-fibers were attempted to apply to functional materials, ${ }^{14-16}$ but they are not optimistic in present state in structural materials and engineering materials owing to very high cost and inert surface. Organic nano-fibers are still kept in laboratory and also poor in temperature-resistance. ${ }^{17}$

In our world, there are some special inorganic mines, which consist of numerous fibril crystals with nano-diameters and certain aspect ratio, such as paly-

${ }^{\dagger}$ To whom correspondence should be addressed (Tel: +86-10-6443-4860, Fax: +86-10-6443-3964, E-mail: zhanglq@ mail.buct.edu.cn). 
gorskite or attapulgite (as defined AT) etc. ${ }^{18,19}$ It is very cheap and rich stored in China and USA. ${ }^{20}$ Chemical formula of AT is $\mathrm{Mg}_{5}[\mathrm{Al}] \mathrm{Si}_{8} \mathrm{O}_{20}(\mathrm{HO})_{2}$ $\left(\mathrm{OH}_{2}\right)_{4} \cdot 4 \mathrm{H}_{2} \mathrm{O}$ and its microstructure can be classified as three levels. ${ }^{21,22}$ Fibrillar single crystal is the smallest structure unit with a length of 500-2000 nm and a diameter of $10-30 \mathrm{~nm}$. Each single crystal consists of many unit layers. Each unit layer consists of two silicone-oxygen tetrahedron. In between adjacent unit layers are five aluminium-oxygen tetrahedron. Unit layers are connected by oxygen atoms and formed a tunnel like crystal structure. Single crystals compactly arrange in parallel and form crystal bundles. These crystal bundles are then agglomerated into micro-scale AT particles. There are a lot of hydroxyls on the surface of AT single crystals. ${ }^{23,24}$ If the suitable technique is developed to exfoliate them into polymer matrix as short nano-fibers, the low cost, high modulus, high surface activity, extremely high temperatureresistance of nano-fibrils will excellently fabricate the need of nano-fiber polymer composites in structural materials field. Junfeng Rong et $a l .{ }^{25}$ have prepared fibril silicate (palygorskite)/PE nanocomposites through in situ polymerization method, where Ziegler-Natta catalyst was first supported on the surface of silicate nanowhiskers to subsequently initiate the polymerization of ethylene on the surface of these nanowhiskers, and as a result nano-dispersion and good mechanical properties of composites were achieved.

In this paper, authors successfully created a series of fibril silicate/rubber nanocomposites by a traditional mechanical mixing technique, combined with an in situ modifying method, where the numerous single crystals with nano-diameter contained in AT micropowder were released to form short nano-fiber/rubber nanocomposites. All these fibril silicate/rubber nanocomposites exhibit excellent mechanical properties, good processing performance, well orientability and low cost.

\section{EXPERIMENTAL}

\section{Materials}

Palygorskite (AT, 1250 mesh) was available from Dalian Global Mineral Co., China. PA66 fiber (Diameter of $20 \mu \mathrm{m}$ and length of $1 \mathrm{~mm}$ ) was bought from Fujin rubber Ltd. Co. of Heilongjiang province, China. Natural rubber (NR, SMR 1\#) was purchased from Yunnan farming cultivation Co. of Yunnan province, China. Ethylene-propylene ethylidene norbornene terpolymer (EPDM33) and acrylonitrile butadiene rubber (NBR41) were produced by JSR Company of Japan; Carboxylated acrylonitrile butadiene rubber (CNBR506) was produced by Nandi Company of Taiwan province, China; Styrene butadiene rubber (SBR1502) was produced by Jihua synthesis rubber factory of China; Chloroprene rubber Neoprene-W (CR) was produced by Dupont Co., USA; Hydrogenated acrylonitrile butadiene rubber (HNBR, Zetpol 2010L, 36.2\%wt ACN content) was purchased from Zeon Co., Japan. Modifier: $\gamma$ (methacryloxy)proxyltrimethoxy silane (KH570, $\mathrm{CH}_{2} \mathrm{C}\left(\mathrm{CH}_{3}\right) \mathrm{COOCH}_{2} \mathrm{CH}_{2}$ $\left.\mathrm{CH}_{2} \mathrm{Si}\left(\mathrm{OCH}_{3}\right)_{3}\right)$, and Bis(3-triethoxysilylpropyl) tetrasulfide $\left(\mathrm{Si} 69,\left(\mathrm{C}_{2} \mathrm{H}_{5} \mathrm{O}\right)_{3} \mathrm{Si}\left(\mathrm{CH}_{2}\right)_{3} \mathrm{~S}_{4} \mathrm{Si}\left(\mathrm{CH}_{2}\right)_{3}\left(\mathrm{OC}_{2} \mathrm{H}_{5}\right)_{3}\right)$ were bought from Shuguang chemicals company of Nanjing, China. Other chemical agents were purchased from chemical store.

\section{Sample Preparation}

Before its utilization, AT was purified in order to remove the impurities such as quartz and carbonate. Firstly, mix 3\% polyacrylate sodium water solution with $30 \%$ AT water suspension, stir the mixture for some time, and then centrifuge the mixture to remove the bottom deposit, finally dry the left water suspension and grind the dried item to get the purified AT.

By a traditional mechanical mixing technique, combined with an in situ modifying method, i.e., the silane coupling agent is directly incorporated into rubber matrix to modify the surface of AT during mixing. Heat two-roll mill to $120-140{ }^{\circ} \mathrm{C}$ and adjust two rolls to smallest distance, rubber and antioxidant 1010 were then put into two-roll mill. Modifying agent and AT together were introduced when rubber became fluidic. After mix them for $5 \mathrm{~min}$ and cool to below $40^{\circ} \mathrm{C}$, active agent (Zinc oxide and stearic acid), accelerator (accelerator D: diphenyl guanidine, accelerator DM: dibenzothiazole disulfide, and accelerator TT: tetramethyl thiuram disulfide) and vulcanizing agent (sulfur) were added thereafter (See Table I) into NR, SBR, EPDM, NBR, CNBR or HNBR matrices. In $C R$ matrix, magnesium oxide was used vulcanizing agent, meantime accelerator NA-22 (Ethylene thiourea) was introduced. The AT loading level is $40 \mathrm{~g} /$ $100 \mathrm{~g}$ rubber without extra description. Thus the compound was finally obtained and sliced at $1.5 \mathrm{~mm}$ tworoll space to prepare for vulcanization. Cross-linking time of the compound was determined from oscillating disc rheometer and the compound was vulcanized at platen presser with 25 tons pressure to get the com-

Table I. Compositions of materials

\begin{tabular}{ll} 
Rubber/g & 100 \\
Antioxidant 1010/g & 2 \\
Active agent/g & $3-6$ \\
Vulcanizing agent and accelerator/g & $3-10$ \\
AT/g & 40 \\
Silane coupling agent/g & $0 / 2.4 \mathrm{phr}$ \\
\hline
\end{tabular}


posite. Test specimens were then tailored from the composite by gauged die. The vulcanization temperature of SBR and NR was $150^{\circ} \mathrm{C}$ and $143^{\circ} \mathrm{C}$ individually, while that of the others was $160^{\circ} \mathrm{C}$.

\section{Characterization}

Tensile test, tear test and hardness of composites are carried out according to ASTM D412, ASTM D624 and ASTM D2240 respectively. During tensile test and tear test, five specimens are needed to give the average value. During the hardness test, testing at three different spots of the test sample is required to give the average value.

Mooney viscosity of the compound is tested on Monsanto Mooney viscosity tester.

A H-800 Transmission Electronic Microscope (TEM) is used to observe the thin section cut from the composites by microtome at $-100^{\circ} \mathrm{C}$, and the nano-fibers included in palygorskite. Acceleration voltage is $200 \mathrm{kV}$. A CAMBRIGE S-250MK3 Scanning Electronic Microscope (SEM) is used to observe the sizes of as-received palygorskite agglomerates. Tensile fractured surface of nanocomposites was observed under environmental scanning electronic microscope (FEI ESEM XL-30).

\section{RESULTS AND DISCUSSION}

\section{Structure}

As is shown in Figure 1a, as-received palygorskite mineral powders are particulates with diameters of $2-5 \mu \mathrm{m}$. Meantime, its fibrils nature can be hazily observed in ESEM photo with higher resolution, shown in Figure 1b. Generally, unpurified AT contain some other inorganic compounds, such as quartz and carbonate. ${ }^{20}$ These chemicals exist in the form of relatively large particles and cannot be dispersed into smaller dispersion units. Their presences in matrix will deteriorate mechanical properties of rubber and therefore must be separated out of AT through a simple purifying procedure (see experimental), as a result AT yield is $65-67 \% .{ }^{26}$ When purified AT particles are dispersed in water by ultrasonic vibration or strong stir, they are dramatically and rapidly separated into lots of nano-fibrils with about $30 \mathrm{~nm}$ in diameter and less than $1500 \mathrm{~nm}$ in length (Figure 1c). Figures 1d1f show the typical microstructure of AT/rubber composites prepared by a traditional mechanical mixing technique, combined with an in situ modifying method. This clearly illustrates that all the palygorskite particulates almost are separated into numerous fibrils with nano-diameter in rubber matrices (styrene butadiene rubber (SBR) and NBR are only taken as two examples). The aspect ratio of nano-fibril is roughly estimated to be 5-30. It is also indicative of strong bonding between these fibrils and rubber as shown in Figure 1e. Consequently, these novel composites are actual short fiber reinforced rubber (namely SFRC) and also rubber based nanocomposites. As a comparison, Figures $1 \mathrm{~g}-1 \mathrm{~h}$ presents the dispersion morphology of AT/SBR composites prepared by directly blending method without the in situ modification. This to say, no silane coupling agent was introduced during blending process. Obviously, the dispersion of AT in SBR is pretty poor and some particulates or nano-fiber bundles with large sizes are observed. Some cavities are also observed on the tensile fractured surface of the composite due to pull out of nano-fiber bundles from rubber matrix. This implied that the interfacial interactions between rubber and AT dispersion units are very weak in the absence of silane coupling agent. Surprisingly, some nano-sized palygorskite dispersions are still observed. This indicates that the interaction between nano-fibrils among palygorskite particles is relatively weaker than that between two layers of clay particles, which is a well-known precursor for preparation of layered silicate nanocomposites. ${ }^{1,2}$ Also, it was proved by the author's group that layered silicate couldn't be exfoliated into nano-dispersion units when it was blended with rubber by the same processing method.

It is a very important characteristic that the interactions between single crystals in AT are much smaller than those between layers in layered silicates. Unlike layer-layer interactions existed in layered silicates, the interaction between AT single crystals is extremely small due to a similar line-line contact and the existence of only weak van der Waals force or hydrogen bond. Furthermore, there are a lot of interstice spaces between these geometrically agglomerated nano-scale single crystals. ${ }^{21}$ These characteristics will facilitate the decohesion (separation) of AT micro-agglomerates into smaller scale crystal bundles and single crystals in water or by surface chemical modification. Besides, this unexpected result is attributed to the good modification of crystal surface by silane coupling agent ${ }^{23}$ and high shear stress on dispersion associated with high viscosity of rubber matrix. Authors suggest that the dispersion of AT in rubber matrix obey the erosion mechanism, ${ }^{27}$ i.e., during mechanical mixing, the siliane coupling agent firstly modify the outer layer of AT dispersion, which weakens the interactions between fibril crystals and disjoints the fibril crystals located in outer layer. Consequently, fibril crystals are exfoliated from dispersed AT particles from outer layer to inner till AT particles completely disappears. Authors also suggest that the looser stacking of fibril silicate be partly responsible for the success of in situ modification method compared with layered silicate. 

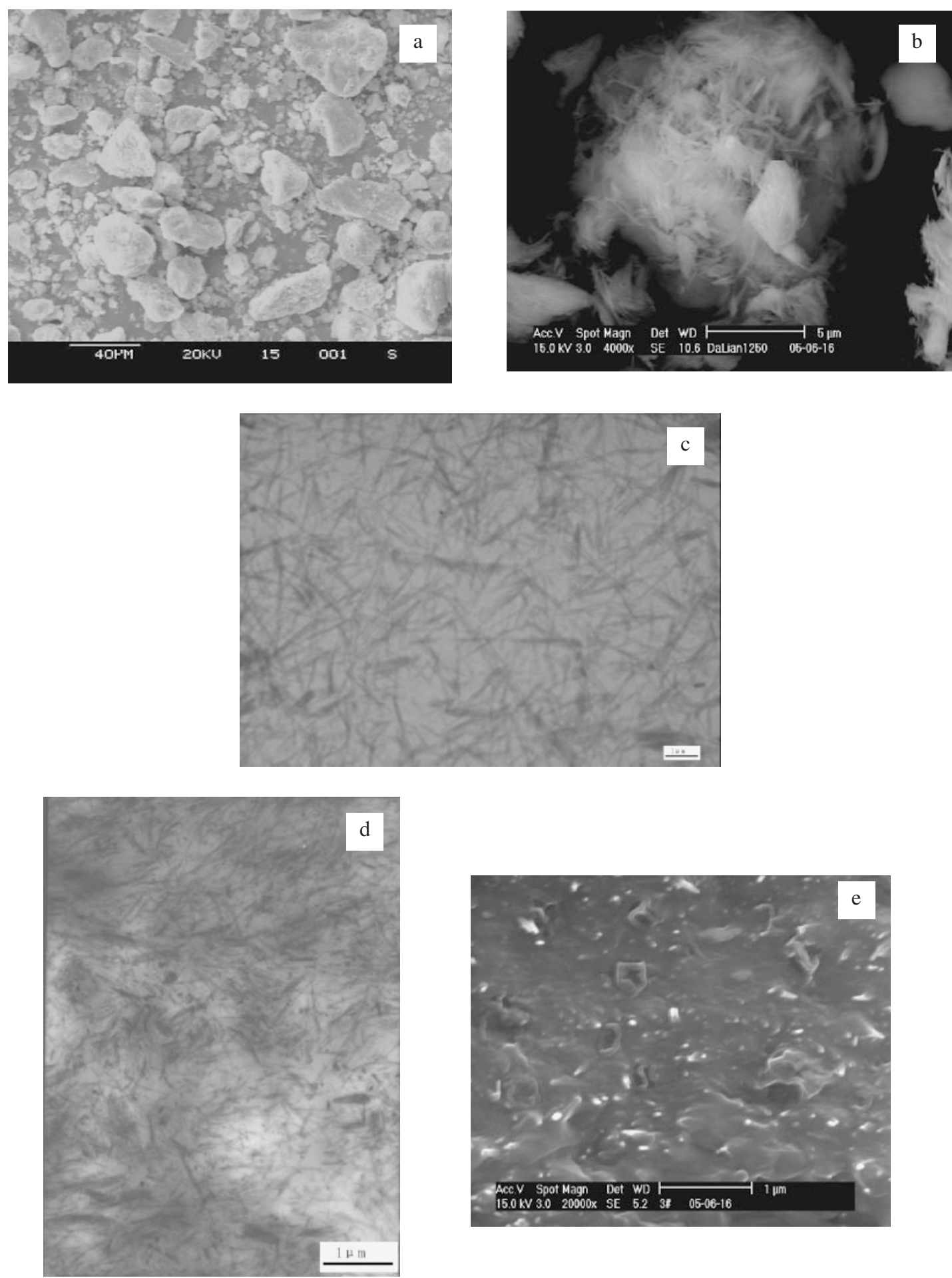

Figure 1. Continued on next page.

\section{Properties}

Silane coupling agent linked onto the surface of nano-fibril silicates during mixing will react with rubber to form a chemical bonding between filler and rubber during crosslinking of rubber, which was proved by nuclear magnetic resonance spectrum. ${ }^{23}$ Dynamic Mechanical Thermal Analysis (DMTA) is generally employed to evaluate the interface change of composites. Here we purposely chose the simpler composites vulcanized by dicumyl peroxide to prepare the sample for DMTA test. Figure 2 shows that the incorporation of palygorskite into rubber matrix greatly weakens the viscoelasticity of rubber in glassy transition region and therefore markedly lowers the loss factor $(\operatorname{Tan} \delta)$ of rubber in this region, while storage modulus $\left(E^{\prime}\right)$ of rubber is remarkably improved. What's more, with the help of silane coupling agent, palygorskite produces the stronger restricting effect to macromolecules 

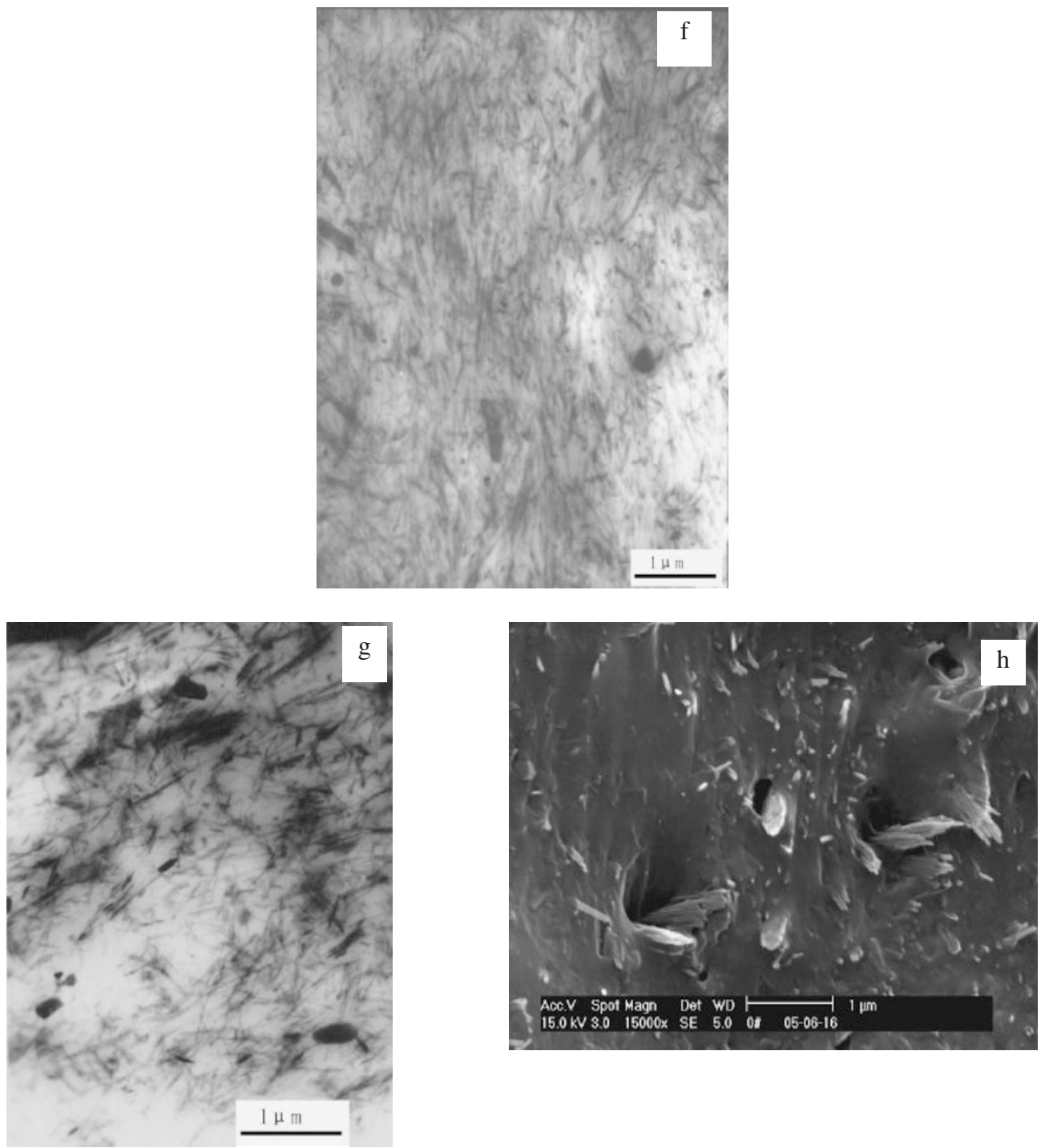

Figure 1. The typical microstructures of AT (a, b, c), AT/SBR nano-composites (d, e); AT/NBR nano-composites (f), AT/SBR microcomposites by melt blending without in situ modification $(\mathrm{g}, \mathrm{h})$.

motion than unmodified palygorskite does due to the finer dispersion and stronger interfacial interaction. ${ }^{23}$ Also, $\gamma$-(methacryloxy)proxyltrimethoxy silane KH570 containing double bond shows a much more intense effect on slowing down the thermal motion of rubber macromolecules than bis(3-triethoxysilylpropyl) tetrasulfide Si69 containing sulfhydryl group. In fact, this effect of silane coupling agent is associated with what is used as vulcanizing agent. Silane coupling agent Si69 should be preferentially chosen when sulfur is extensively used as vulcanizing agent in industry. ${ }^{23}$ In the following discussion, Si69 was used as modifier unless additional description in that sulfur contained in both these accelerators or vulcanizing agents and Si69 took part in the crosslinking reaction of rubber.

The most important mechanical characteristics of SFRC should be high modulus and apparent anisotropy. Figure $3 a$ shows the stress-strain behaviors of var- ious kinds of filler reinforced NBR composites. PA66 short fiber is a kind of micro-fiber, while nano-carbon black N330 is a kind of particulate filler that have been extensively used in rubber industry for many years. Obviously, AT/NBR nanocomposites exhibit much higher modulus, higher stress than AT/NBR composites by directly blending without in situ modification, and traditional nano-carbon black/NBR nanocomposites at the same strain, approaching to stress-strain characteristics of PA66 short fiber reinforced NBR composites. Although the modulus of AT/NBR nanocomposite is slightly lower than that of PA66 short fiber/NBR composites, the gentler linear stress-strain behavior displayed by AT/NBR nanocomposite is more required in rubber industry. In addition, the highest tensile strength of AT/NBR nanocomposites distinguishes it and favors many application fields. Compared with short micro fiber, nano-fiber decreases the stress concentration existed 

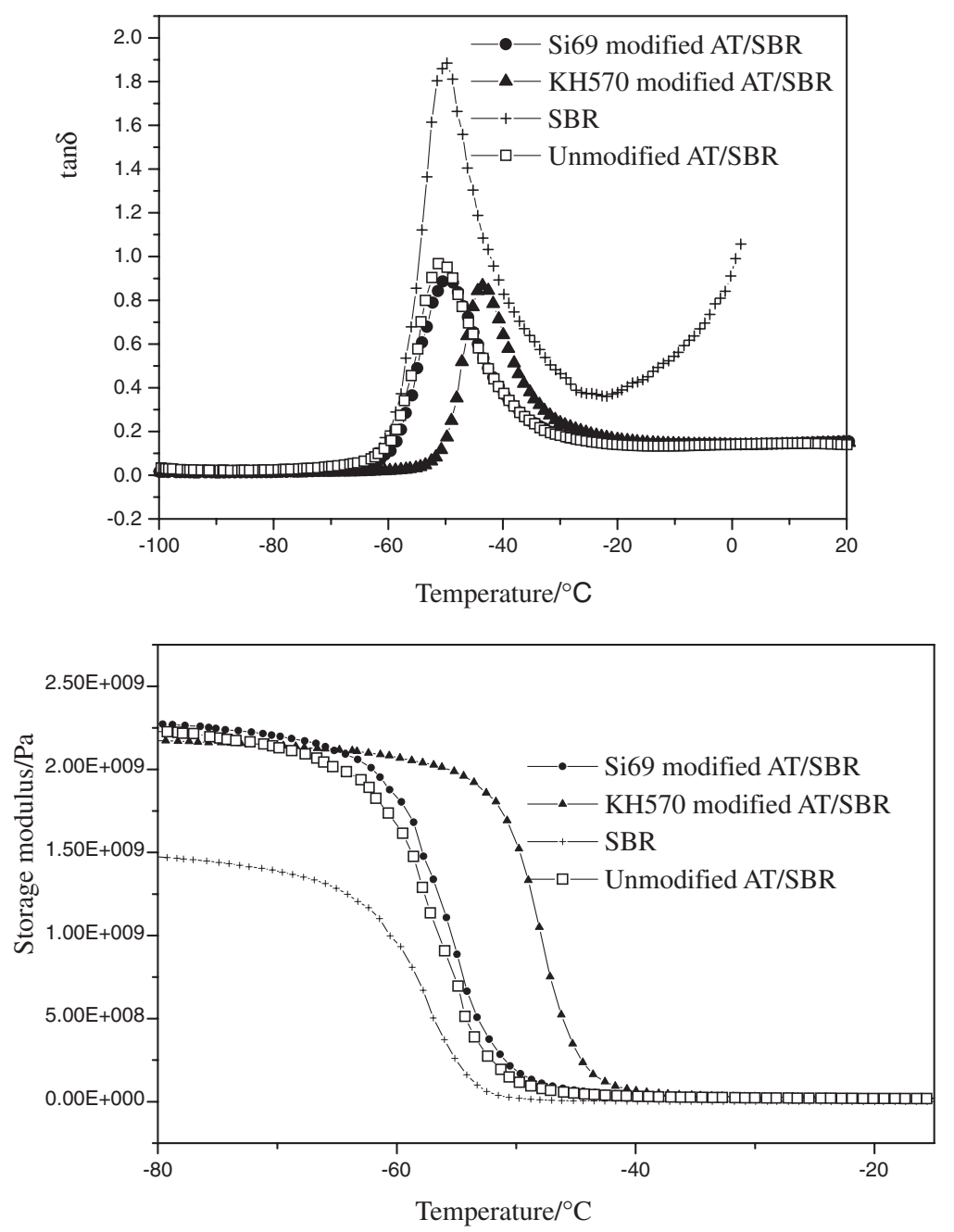

Figure 2. Dynamic properties of palygorskite/SBR with different silane coupling agent.

in composites and increases the interfacial area but decreases the stiffness of dispersion unit that is correlated with the cubic of dimension of dispersion. Figure $3 b$ reveals the comparison of Mooney viscosity between nano-fiber and micro-fiber filled acrylonitrile butadiene rubber (NBR) compounds (unvulcanized). The temperature for Mooney viscosity is purposely set at $40^{\circ} \mathrm{C}$ in order to avoid PA66 fiber from softening. It is obvious that AT/NBR compound with lower Mooney viscosity presents better flow property at the same fiber volume fraction mainly owing to the smaller absolute length of nano-fibrils. Furthermore, the extrusion appearance of AT/NBR compound from capillary rheometer is pretty smooth compared with that of short nylon fiber/NBR compound.

Table II summarizes mechanical properties of various rubber based short nano-fibrils silicate nanocomposites made by a traditional mechanical mixing technique, combined with an in situ modifying method. It strongly reveals that nano-fibril silicates can endow various rubbers with excellent performances, such as SBR, NBR, NR, EPDM, CNBR, HNBR and CR.
Compared with neat rubber, the corresponding AT/ rubber nanocomposites presented remarkable increase in hardness, stress at 100\% strain, tensile strength and tear strength, as well as decrease in elongation at break. By way of exception, tensile strength of AT/ NR nanocomposite is a little lower than that of neat NR. It is likely that the immobility of rubber macromolecules resulted from strong bonding between AT and matrix impairs the crystallization of NR due to the orientation of macromolecules at large tensile strain. Still, tear strength and stress at $100 \%$ strain of AT/NR composite are increased by times, indicating the excellent reinforcement of AT. It should be pointed out, the excellent properties of AT/rubber nanocomposites are not only related to the nanodispersion of AT in rubber matrix, but also attributed to the strong interfacial interactions between nanofibril silicate and rubber, which benefits from chemical bonding of silane coupling agent and those active hydroxyl groups located on the surface of fibril silicate crystals. ${ }^{23}$

Figure 4 shows the effect of palygorskite concen- 

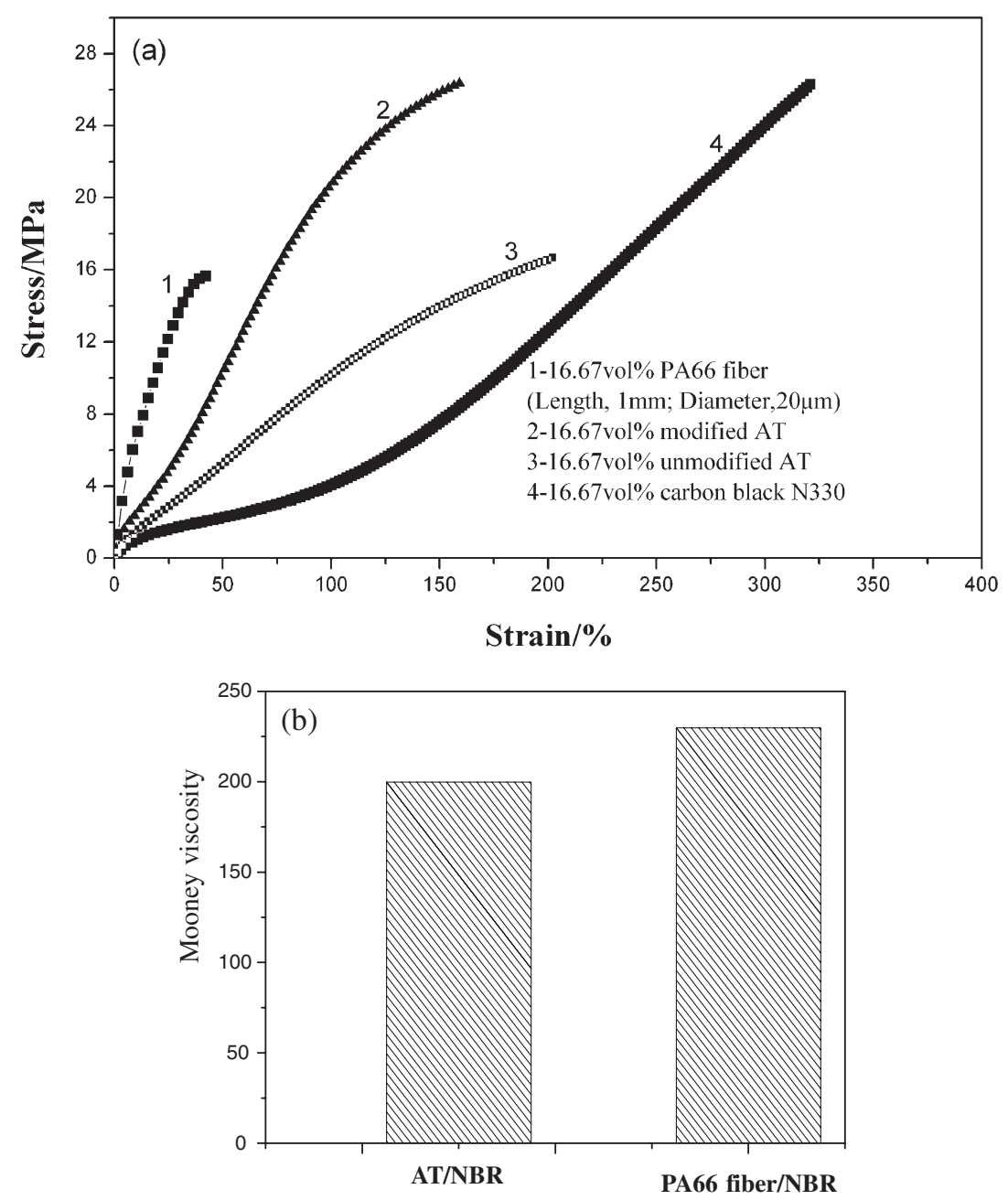

Figure 3. Stress-strain characteristics (a) and Mooney viscosity (b) of various kinds of filler reinforced NBR composites.

Table II. The mechanical properties of palygorskite/rubber nanocomposites*

\begin{tabular}{|c|c|c|c|c|c|c|c|c|c|c|c|c|c|c|}
\hline Properties & SBR & $\begin{array}{c}\mathrm{AT} \\
/ \mathrm{SBR}\end{array}$ & NR & $\begin{array}{l}\mathrm{AT} \\
/ \mathrm{NR}\end{array}$ & NBR & $\begin{array}{c}\mathrm{AT} \\
/ \mathrm{NBR}\end{array}$ & CNBR & $\begin{array}{c}\mathrm{AT} \\
/ \mathrm{CNBR}\end{array}$ & EPDM & $\begin{array}{c}\mathrm{AT} \\
/ \mathrm{EPDM}\end{array}$ & HNBR & $\begin{array}{c}\mathrm{AT} \\
/ \mathrm{HNBR}\end{array}$ & CR & $\begin{array}{l}\mathrm{AT} \\
/ \mathrm{CR}\end{array}$ \\
\hline $\begin{array}{l}\text { Shore A } \\
\text { hardness }\end{array}$ & 45 & 73 & 41 & 74 & 54 & 83 & 61 & 72 & 70 & 78 & 57 & 80 & 51 & 83 \\
\hline $\begin{array}{l}\text { Stress at } 100 \% \\
\text { strain (MPa) }\end{array}$ & 0.8 & 7.6 & 1.0 & 9.1 & 1.2 & 12.1 & 2.3 & 8.3 & 1.4 & 7.0 & 1.3 & 28.6 & 1.1 & 13.8 \\
\hline $\begin{array}{l}\text { Tensile strength } \\
\text { (MPa) }\end{array}$ & 2.3 & 14.7 & 29.3 & 24.5 & 3.2 & 22.7 & 9.7 & 22.7 & 4.7 & 10.7 & 6.1 & 30.4 & 6.6 & 14.6 \\
\hline $\begin{array}{l}\text { Elongation at } \\
\text { break }(\%)\end{array}$ & 556 & 344 & 660 & 390 & 308 & 290 & 412 & 237 & 356 & 177 & 270 & 109 & 509 & 113 \\
\hline $\begin{array}{l}\text { Tear strength } \\
(\mathrm{kN} / \mathrm{m})\end{array}$ & 11.6 & 60.8 & 30.3 & 65.4 & 14.3 & 58.8 & 22.9 & 48.5 & 18.1 & 36.1 & 13.9 & 26.4 & no data & no data \\
\hline
\end{tabular}

*The AT loading level is $40 \mathrm{~g} / 100 \mathrm{~g}$ rubber.

tration on mechanical properties of palygorskite/NBR composites. As the palygorskite concentration ascends, the stress at $100 \%$ strain and tensile strength of the nanocomposite remarkably mount up whereas its elongation at break falls down as well. Due to the small dimension of nano-fibril, the palygorskite loading level can reach up to $100 \mathrm{~g} / 100 \mathrm{~g}$ rubber, which is very conducive to reducing the cost of com- posites. Surprisingly, at the moment tensile strength of the nanocomposite surprisingly exceeds $41.7 \mathrm{MPa}$, which is hard for nano-carbon black reinforced NBR composite to reach. This mechanical characteristic is very promising for structural application of rubber materials.

Generally, the stress difference at certain strain and tensile Young's modulus difference of SFRC between 


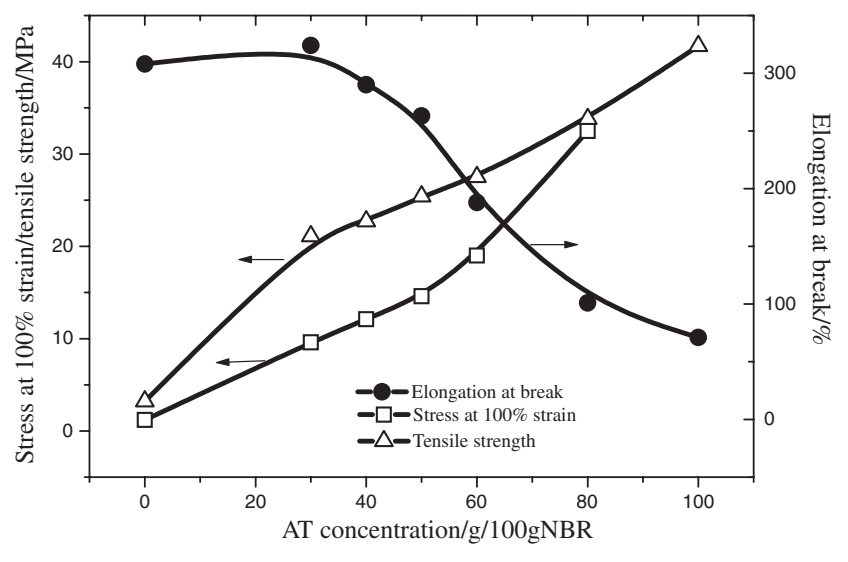

Figure 4. The effect of AT concentration on mechanical properties of AT/NBR composites.

the two directions, i.e., parallel (L direction) and perpendicular ( $\mathrm{T}$ direction) to fiber orientation can well reflect the anisotropy of the composite. In order to make nano-fibrils oriented in rubber matrix, simulating the industrial way of orienting the SFRC, we adjusted two rolls of mixing miller to the smallest interval so as to produce the maximal shear. Figure 5a presents that both the tensile Young's modulus difference $\left(E_{\mathrm{L}}-E_{\mathrm{T}}\right)$ and the stress difference $\left(\sigma_{\mathrm{L}}-\sigma_{\mathrm{T}}\right)$ at $100 \%$ strain of the AT/NBR rise up as the concentration of palygorskite increases. At lower concentration, short nano-fibril silicates don't tend to orientate mainly because of their low absolute length. Figure 5b clearly expresses the evident orientation of nano-fibril silicates at a very high loading ( $80 \mathrm{~g} / 100 \mathrm{~g}$ NBR). It is wonderful that the dispersion and separation of AT is still good at such a high loading. As discussed above, the unique structure of AT resulted in the simplicity and efficiency in nano-dispersing besides the effect of silane coupling agent.

\section{CONCLUSIONS}

In summary, a series of novel nano-fibril silicate/ rubber nanocomposites were prepared by using a traditional mechanical mixing technique, combined with an in situ modifying method. These nanocomposites exhibit excellent mechanical characteristics that are similar to that of short fiber reinforced rubber, excellent processing properties as expected, low cost and easily practiced by industry, which hence presents very bright future in rubber industry.

Acknowledgment. The authors thank High Technology Plan of Ministry of Science and Technology of China (2002AA334050, and 2003AA333060) for capitals as well as Beijing Municipal New Star Project of China (H020820620120).

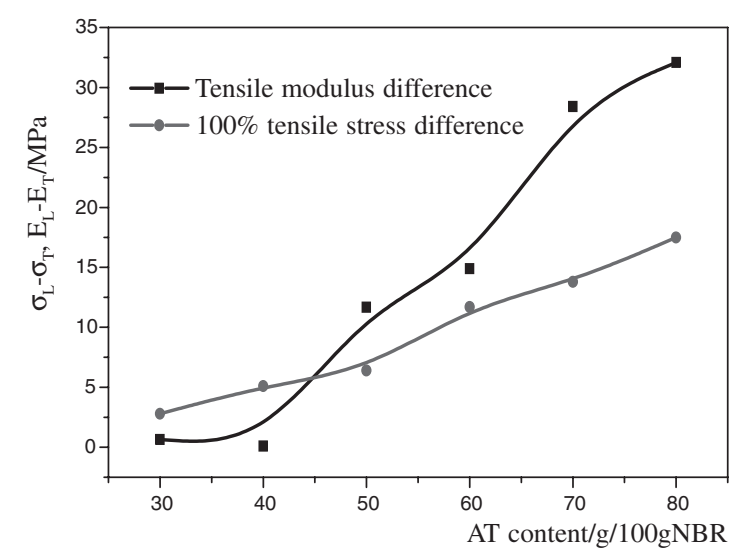

(a)

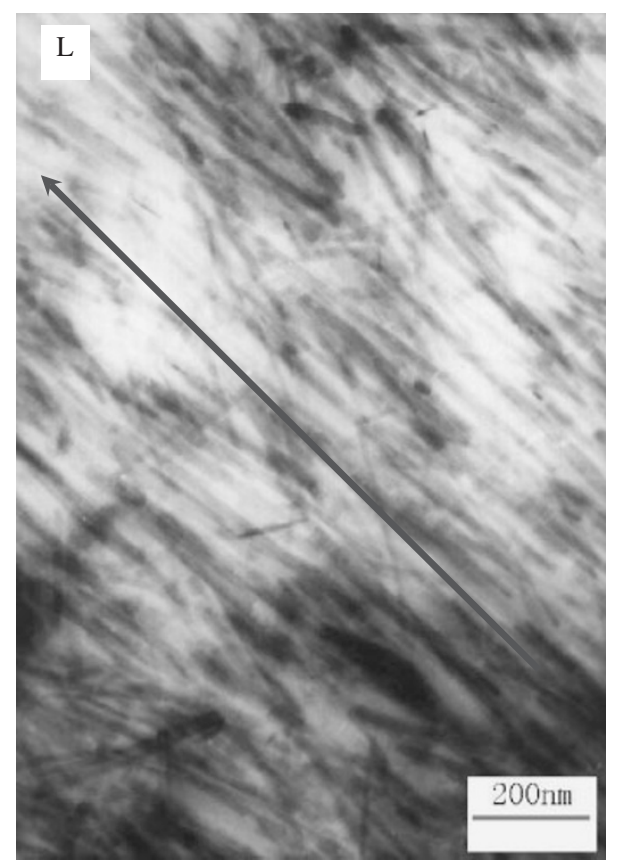

(b) $80 \mathrm{~g} \mathrm{AT} / 100 \mathrm{~g}$ NBR

Figure 5. The anisotropy of AT/NBR composites.

\section{REFERENCES}

1. E. P. Giannelis, Adv. Mater., 8, 29 (1996).

2. R. A. Vaia, E. P. Giannelis, et al., J. Am. Chem. Soc., 117, 7568 (1995).

3. A. Okada, M. Kawasumi, T. Kurauchi, and O. Kamigaito, Polym. Prepr. (Am. Chem. Soc., Div. Polym. Chem.), 28, 447 (1987).

4. J. Karger-Kocsis and C. M. Wu, Polym. Eng. Sci., 44, 1083 (2004).

5. T. Kurauchi, A. Okada, T. Nomura, T. Nishio, S. Saegusa, and R. Deguchi, SAE Technical Paper Ser., 910584 (1991).

6. S. S. Ray and M. Okamato, Prog. Polym. Sci., 28, 1539 (2003)

7. P. Ajayan, O. Astephan, C. Colliex, and D. Trauth, Science, 264, 1212 (1994).

8. D. B. Romero, M. Carrard, W. H. de Heer, and L. Zuppiroli, Adv. Mater., 8, 899 (1996). 
9. Z. X. Jin, K. P. Pramoda, G. Q. Xu, and S. H. Goh, Chem. Phys. Lett., 337, 43 (2001).

10. K. Lozano and E. V. Barrera, J. Appl. Polym. Sci., 79, 125 (2001).

11. Y. L. Yang, M. C. Gupta, K. L. Dudley, and R. W. Lawrence, Adv. Mater., 17, 1999 (2005).

12. J. Fan, M. Wan, D. B. Zhu, Z. W. Pan, and S. S. Xie, J. Appl. Polym. Sci., 74, 2605 (1999).

13. Y. R. Liang, Y. Q. Wang, Y. P. Wu, and L. Q. Zhang, Polym. Test., 24, 12 (2005).

14. O. Regev, P. N. B. Elkati, J. Loos, and C. E. Koning, Adv. Mater., 16, 248 (2004).

15. S. A. Curran, P. M. Ajayan, W. J. Blau, and D. L. Carroll, Adv. Mater., 10, 1091 (1998).

16. H. Ago, K. Petritsch, M. S. P. Shaffer, A. H. Windle, and R. H. Friend, Adv. Mater., 11, 1281 (1999).

17. H. Fong and D. H. Reneker, J. Polym. Sci., Part B: Polym. Phys., 37, 3488 (1999).

18. G. E. Vanscoyoc, C. J. Serna, and J. L. Ahlrichs, Am.
Mineral., 64, 215 (1979).

19. M. Tian, L. J. Chen, W. L. Liang, and L. Q. Zhang, Macromol. Mater. Eng., 290, 681 (2004).

20. S. H. Yan, "Clay Mine," Beijing Geology Press, China, 1981, p. 317.

21. J. Gu, N. Liu, Y. Li, and Y. J. Ma, J. Silicate (China), 6, 50 (1999).

22. H. Murray Haydn, Appl. Clay Sci., 17, 207 (2000).

23. M. Tian, W. L. Liang, G. Y. Rao, L. Q. Zhang, and C. X. Guo, Compos. Sci. Technol., 65, 129 (2005).

24. C. J. Serna, G. E. Vanscoyoc, and J. L. Ahlrichs, Am. Mineral., 62, 784 (1977).

25. J. F. Rong, Z. H. Jing, H. Q. Li, and M. Sheng, Macromol. Rapid Commun., 22, 329 (2001).

26. M. Tian, C. D. Qu, Y. X. Feng, and L. Q. Zhang, J. Mater. Sci., 38, 4917 (2003).

27. S. P. Rwei, I. Manas-Zloczower, and D. L. Feke, Polym. Eng. Sci., 31, 558 (1991). 